\title{
Safety and efficacy of argon plasma coagulator ablation therapy for flat colorectal adenomas
}

\author{
A. García, O. Núñez, C. González-Asanza, A. Parera, L. Menchén, C. Ripoll, C. Senent, E. Cos and \\ P. Menchén
}

Unit of Gastrointestinal Endoscopy. Department of Digestives Diseases. Hospital Gregorio Marañón. Madrid, Spain

\begin{abstract}
Introduction: argon-plasma coagulation (APC) has been used safely and efficaciously in multiple settings including colon polyp treatment. The aim of this study was to evaluate APC efficacy and safety in the treatment of flat colorectal adenomas.

Materials and methods: APC ablation was prospectively performed and evaluated in 22 consecutive patients with colorectal adenomas, 11 of which had large sessile adenomas that were treated with piecemeal polypectomy and APC ablation of residual adenomatous tissue, whereas the remaining eleven patients with flat or carpet-like adenomas were only treated with APC. The mean initial longitudinal extension of adenomas to be treated with APC was $22 \mathrm{~mm}$ (range, 20 to $40 \mathrm{~mm}$ ).

Results: the mean age of patients was 70 years. Adenomas were found most frequently in the rectum (50\%) and cecum (23\%). Complete ablation was achieved in $90.9 \%$ of adenomas. Recurrence was observed in $20 \%$ of patients, all of them in the rectum, after a mean follow-up period of 16.3 months (range, 8 to 35). All recurrences were managed satisfactorily. No major complications were seen.

Conclusions: argon plasma coagulator ablation of flat colorectal adenomas is an efficacious and safe technique, specially in the right colon, but results must be confirmed in controlled trials with a higher number of patients.
\end{abstract}

Key words: Argon-plasma coagulation. Colorectal adenomas.

García A, Núñez O, González-Asanza C, Parera A, Menchén L, Ripoll C, Senent C, Cos E, Menchén P. Safety and efficacy of argon plasma coagulator ablation therapy for flat colorectal adenomas. Rev Esp Enferm Dig 2004; 96: 315-321.

Recibido: $10-04-03$

Aceptado: 15-12-03.

Correspondencia: Óscar Núñez Martínez. Servicio de Aparato Digestivo. Hospital General Universitario Gregorio Marañón. C/ Dr. Esquerdo, 46. 28007 Madrid. Telf.: 9158683 07. Fax: 9142650 24. e-mail: onumar@inicia.es

\section{INTRODUCTION}

Argon-plasma coagulation (APC) has been used in digestive endoscopy since 1991, when a specific electrode that could be introduced through the endoscope channel was designed (1). It is a monopolar electrosurgical device that does not need physical contact with tissue, as a highfrequency electrical current is transmitted to the tissue by ionized argon gas. APC produces superficial thermal damage about 2-3 $\mathrm{mm}$ deep depending on energy output and the time current is applied (2).

Endoscopic APC has been applied in different settings such as: localized or diffuse vascular lesions like angiodysplasia or watermelon stomach $(3,4)$, Barrett's esophagus $(5,8)$, radiation colitis $(9-11)$, bleeding peptic ulcer $(4,12)$, Zenker's diverticulum (13), malignant tumors and specially in repermeabilization of intraluminal growths after prosthesis placement for the palliative treatment of esophagus cancer (14-16) and colonic polyps $(3,4)$.

Large sessile polyps are more likely to have neoplastic foci, with a high rate of recurrence after excision in previous series (16-46\%), as well as a higher frequency of postpolypectomy complications such as bleeding and perforation (17-19). For these reasons, alternative ways of destroying residual tissue with a lower percentage of complications have been researched, usually associated with piecemeal polypectomy. Diverse results have been reported on the use of argon plasma coagulation in this setting (2,20-22).

The aim of this study was to analyze the efficacy of APC ablation therapy for flat and sessile polyps in association or not with previous piecemeal polypectomy in our Endoscopy Unit, and to evaluate the safety of this therapy in clinical practice.

\section{MATERIAL AND METHODS}

Thirty-five patients with flat or sessile polyps treated with APC from May 1997 to December 2000 were included 
in this study. In this group, 13 patients were excluded from the analysis because of one of the following reasons: high-grade dysplasia, follow-up shorter than 6 months, and loss to follow-up.

All procedures were performed on an outpatient basis initially. An oral polyethylene glycol electrolyte lavage solution and occasionally cleansing enemas were used for colonic preparation. An informed consent was obtained from each patient before each procedure. Conscious sedation was performed by an anesthetist using intravenous propofol when unsedated colonoscopy was not tolerated. Pulse oximetry was used for monitorization.

A specific device for argon gas application with a $2.5 \mathrm{~L} / \mathrm{min}$ gas flow and variable electric power (from 40 to $80 \mathrm{~W}$ ) was used depending on polyp location (ERBE APC300; ERBE Electromedizin, Tübingen, Germany). APC treatment was administered using a standard single-channel flexible colonoscope (Fujinon 200, Omiya, Japan). The endoscopic polypectomy was performed using a standard snare and predefined electrocautery energy (Endocut; ERBE ICC 200; ERBE Electromedizin, Tübingen, Germany). Adenomatous tissue extension was measured and compared using open biopsy forceps.

APC treatment was scheduled every 15 days until adenomatous tissue had completely disappeared. Afterwards, a colonoscopic review was done first at 6 months and then annually. Colonoscopic examinations were recorded so that polyp localization was rendered easier on follow-up. Biopsies ware taken in the treated area with or without macroscopic adenomatous tissue in each endoscopic revision.

The endoscopic treatment applied was APC in 11 patients with flat or carpet-like adenomas, while the remaining 11 patients with large sessile adenomas were first treated with piecemeal polypectomy using an electrocautery snare and then completed with APC on residual tissue (Fig. 1). We based our definition of flat adenoma on previously proposed criteria $(23,24)$. The mean initial longitudinal extension of the area treated with APC was $22 \mathrm{~mm}$ (20-40). Previous cold forceps biopsies were taken in those patients who were treated only with APC, while the tissue of those polyps partially resected was sent for histological examination.

Complete response was defined as the absence of visible adenomatous tissue both macroscopically and in the histological examination of biopsies taken during endoscopy at follow-up. Recurrence was defined as the presence of new proliferation of adenomatous tissue in areas of previously treated mucosa with documented complete response. Treatment failure was defined as a persistence of a polyp at least half its initial size following three APC sessions.

Qualitative variables expressed as percentages were compared using Fisher's test. Quantitative variables, expressed as means with their range, were compared using
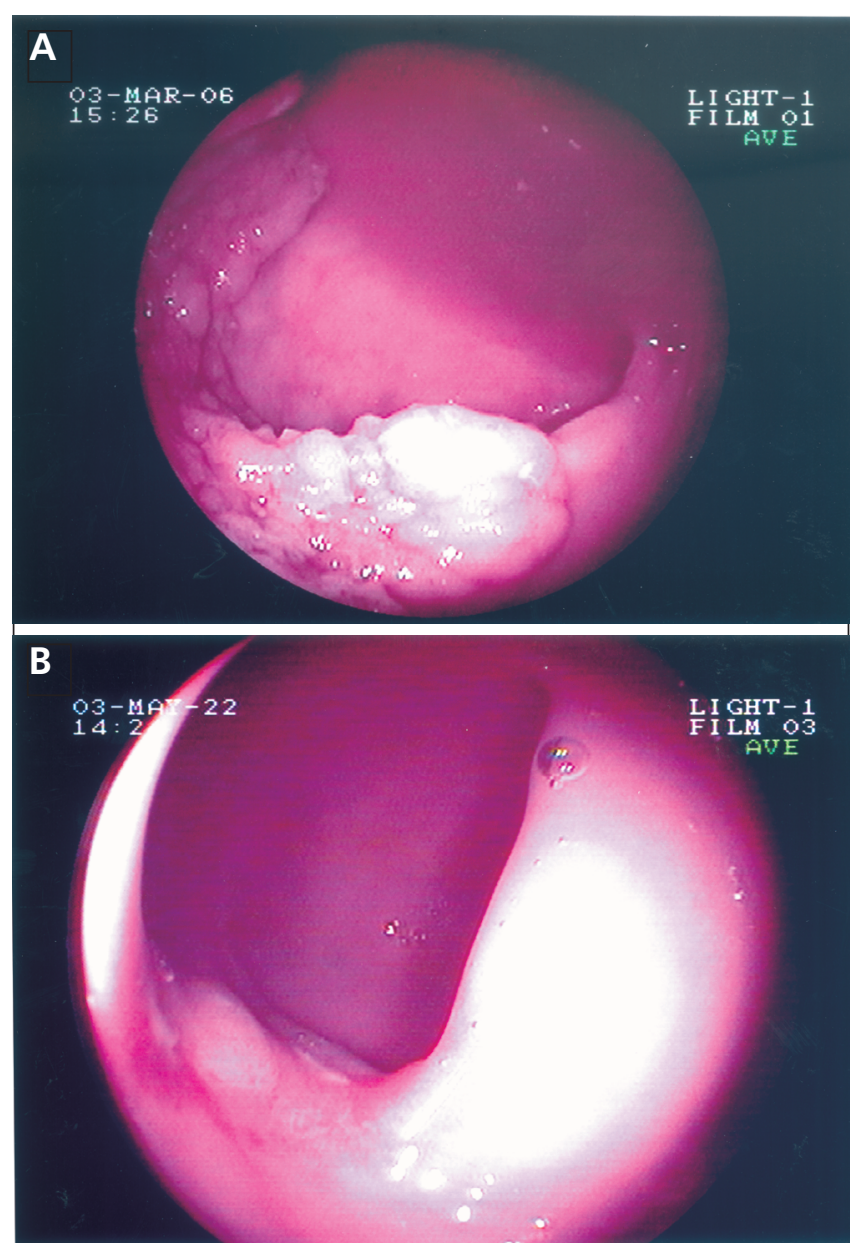

Fig. 1.- Colonoscopic images. Colon adenoma before (A) and after (B) piecemeal polypectomy and argon plasma coagulation ablation therapy. Imágenes procedentes de procedimiento de colonoscopia en las cuales se observa un pólipo sesil rectal antes $(A)$ y después (B) de tratamiento endoscópico con polipectomía fragmentada y AP.

Student's t-test. Differences were considered significant when $p<0.05$. All statistical analyses were performed using the SPSS software, version 10.0.

\section{RESULTS}

Twenty-two patients were included with a mean follow-up period of 15 months (range, 6 to 35). Adenomas localized in the rectum were most frequent $(50 \%)$. The characteristics of patients, colorectal polyps and treatments are indicated in table I.

Histological examination revealed a villous component in $86 \%$, and mild or moderate dysplasia in $81 \%$ of polyps. 
Table I. Baseline characteristics

\begin{tabular}{lc}
\hline Gender (\%) & \\
$\quad$ Male & $14(64)$ \\
Female & $8(36)$ \\
Age $(y r)$ & $70(57-83)$ \\
Adenomatous tissue size $(\mathrm{mm})$ & $22(20-40)$ \\
Site (\%) & \\
Rectum & $11(50)$ \\
Cecum & $5(22.7)$ \\
Left colon & $4(18.2)$ \\
Transverse colon & $2(9.1)$ \\
Number of sessions & $1.7(1-3)$ \\
Follow-up period (months) & $15(6-35)$ \\
Technique (\%) & $11(50)$ \\
APC & $11(50)$ \\
APC and piecemeal polypectomy & \\
\hline
\end{tabular}

Complete response was obtained in $90.9 \%$ of adenomas treated with a mean of 1.7 sessions (range, 1 to 3 ). Two patients who did not respond to APC after 3 sessions were considered treatment failures. One of the polyps in the rectum was $20-\mathrm{mm}$ long and had been treated with only APC, while the other polyp was 40-mm long and had been treated with piecemeal polypectomy initially, with $20 \mathrm{~mm}$ remaining afterwards. Both cases were villous adenomatous polyps with mild dysplasia on histological examination.

Recurrence was detected in 4 patients (20\%) after a mean period of 16.3 months (range, 8 to 35 months). All recurrences were observed in rectal polyps. Three cases were managed endoscopically with satisfactory results, but one patient required endoanal surgical resection.

There was no relationship between recurrence and previous piecemeal polypectomy. Only 1 patient out of 10 $(10 \%)$ who had been treated exclusively with APC recurred, and so did 3 patients $(30 \%)$ who had been treated with both methods $(p>0.05)$. Recurrence was related with the initial size of the adenomatous tissue to be treated. Thus, those that recurred had an initial mean extension of $23.8 \pm 7.5 \mathrm{~mm}$, while those that maintained a complete remission had a size of $13.2 \pm 7 \mathrm{~mm}(\mathrm{p}=0.01)$.

No major complications related with the procedure occurred. One patient, after the first session of APC for a cecal polyp, developed inflammatory polyps in the treated mucosa, which disappeared in the following revisions. Two additional patients suffered from transient abdominal pain, which was managed on an outpatient basis.

\section{DISCUSSION}

Argon-plasma coagulation is based on the transmission of a high-frequency current through argon gas to result in thermal damage. It has been safely and efficaciously used in multiple settings (3-16).

The rational basis for the use of APC in the treatment of colorectal polyps is ablation of residual adenomatous tissue after piecemeal polypectomy of big polyps and stalk hemostasia. Zlatanic et al. (20) studied the recurrence of adenomatous tissue after piecemeal polypectomy, and observed that recurrence occurred in all cases where residual tissue remained, and in 46 and 50\% of cases, respectively, with no residual adenomatous remnants or where APC was applied. In a most recent randomized, controlled study of recurrence prevention after piecemeal resection, APC administration following complete snare excision was seen to reduce polyp recurrence in spite of the small size of the sample (21). When snare excision was not complete and APC was applied, polyp recurrence was observed in 6 out of 13 patients. In another study that was published in abstract form (22), adenomatous tissue recurrence following APC was observed in 3.5\% of patients after a mean follow-up period of 6 months. All studies concluded that residual adenomatous tissue treatment with APC after piecemeal polypectomy is safe and efficacious.

In our study, APC was used as the first and only treatment of flat polyps, as well as for complete ablation of residual adenomatous tissue after piecemeal polypectomy. Recurrence was observed in $20 \%$ of cases after a mean follow-up of 16 months. Although our recurrence rate matches those previously described (3.5-46\%), our followup period was longer. All recurrences were satisfactorily managed endoscopically except in one case, which required endoanal surgery resection.

Although APC was administered as the initial and only treatment of flat adenomatous polyps, this approach did not determine a higher recurrence rate, which was only related with the initial size of the treated lesion. This finding may be relevant particularly in the right colon, where the colonic wall is thinner and therefore more susceptible to perforation. In our study, $22.7 \%$ of polyps treated were located in the cecum, but it was not related with a higher rate of complications.

Other techniques such as laser photocoagulation (25) and endoscopic mucosal resection $(26,27)$ had been already used in this setting with good results, but APC ablation is an easier procedure that is associated with a lower risk of complications, and thus may become a first-choice technique with this indication. However, this hypothesis has still to be evaluated in controlled, randomized clinical trials comparing these modalities of endoscopic treatment for adenoma.

Inflammatory polyps emerged after APC application in one cecal polyp, but they disappeared spontaneously as reported in following controls. This complication had been previously described after APC in the upper gastrointestinal tract (28).

We conclude that the use of APC for the treatment of flat colorectal polyps is safe and efficacious. This finding is specially interesting when the treatment of right-colon and cecum polyps is taken into account, but such results must obtain confirmation in controlled trials with a higher number of patients. 


\section{REFERENCES}

1. Farin G, Grund KE. Technology of argon plasma coagulation with particular regard to endoscopic applications. Endoscop Surg 1994; 2: 71-7.

2. Watson JP, Bennett MK, Griffin SM, et al. The tissue effect of argon plasma coagulation on esophageal and gastric mucosa. Gastrointest Endosc 2000; 52: 342-5.

3. Johanns W, Luis W, Janssen J, et al. Argon plasma coagulation (APC) in gastroenterology: experimental and clinical experiences. Eur J Gastroenterol Hepatol 1997; 9 (6): 581-7.

4. Wahab PJ, Mulder CJ, den Hartog G, et al. Argon plasma coagulation in flexible gastrointestinal endoscopy: pilot experiences. Endoscopy 1997; 29 (3): 176-81.

5. Kochman ML. Eradication of Barrett's mucosa with argon plasma coagulation and acid suppression: immediate and mid-term results. Gastrointest Endosc 1999; 50 (6): 884-6.

6. May A, Gossner L, Gunter E, et al. Local treatment of early cancer in short Barrett's esophagus by means of argon plasma coagulation: initial experience. Endoscopy 1999; 31 (6): 497-500.

7. Grade AJ, Shah IA, Medlin SM, et al. The efficacy and safety of argon plasma coagulation therapy in Barrett's esophagus. Gastrointest Endosc 1999; 50 (1): 18-22.

8. Van den Boogert J, Van Hillegersberg R, Siersema PD, et al. Endoscopic ablation therapy for Barrett's esophagus with high-grade dysplasia: a review. Am J Gastroenterol 1999; 94 (5): 1153-60.

9. Kaassis M, Oberti E, Burtin P, et al. Argon plasma coagulation for the treatment of hemorrhagic radiation proctitis. Endoscopy 2000; 32 (9): 673-6.

10. Morrow JB, Dumot JA, Vargo JJ. Radiation-induced hemorrhagic colitis treated with argon plasma coagulator. Gastrointest Endosc 2000; 51 (4 Pt 1): 498-9.

11. Silva RA, Correia AJ, Dias LM, et al. Argon plasma coagulation therapy for hemorrhagic radiation proctosigmoiditis. Gastrointest Endosc 1999; 50 (2): 221-4.

12. Cipolleta L, Bianco MA, Rotondano G, et al. Prospective comparison of argon plasma coagulator and heater probe in the endoscopic treatment of major peptic ulcer bleeding. Gastrointest Endosc 1998; 48 (2): 191-5

13. Mulder CJ. Zapping Zenker's diverticulum: gastroscopic treatment. Can J Gastroenterol 1999; 13 (5): 405-7.

14. Akhtar K, Byrne JP, Bancewicz J, et al. Argon beam plasma coagula- tion in the management of cancers of the esophagus and stomach. Surg Endosc 2000; 14 (12): 1127-30

15. Watson JP, Bennett MK, Griffin SM, et al. The tissue effect of argon plasma coagulation on esophageal and gastric mucosa. Gastrointest Endosc 2000; 52 (3): 342-5.

16. Grund KE, Storek D, Becker HD. Highly flexible self-expanding meshed metal stents for palliation of malignant esophagogastric obstruction. Endoscopy 1995; 27 (7): 486-94.

17. Binmoeller KF, Bognacker S, Seifert H, et al. Endoscopic snare excision of "giant" colorectal polyps. Gastrointest Endosc 1996; 43: 1838.

18. Waye JD. How big is to big? Gastrointest Endosc 1996; 43: 256-7.

19. Walsh RM, Ackroyd FW, Shellito PC. Endoscopic resection of large sessile colorectal polyps. Gastrointest Endosc 1992; 38: 303-8.

20. Zlatanic J, Waye JD, Kim PS, et al. Large sessile colonic adenomas: use of argon plasma coagulator to supplement piecemeal snare polypectomy. Gastrointest Endosc 1999; 49 (6): 731-5.

21. Brooker JC, Saunders BP, Shah SG, et al. Treatment with argon plasma coagulation reduces recurrence after piecemeal resection of large sessile colonic polyps: a randomized trial and recommendations. Gastrointest Endosc 2002; 55: 371-5.

22. Regula J, Wronska E, Polkwski M, et al. Argon plasma coagulation (APC) after piecemeal polypectomy for colorectal adenomas. Endoscopy 1996; 28: s61-2 (A1274).

23. Sawada T, Hojo K, Moriya Y. Colonoscopic management of focal and early colorectal carcinoma. Balliere's Clinical Gastroenterology 1989; 3: 627-45.

24. Rembacken BJ, Fujii T, Dixon MF, et al. Flat and depressed colonic neoplasms: a prospective study of 1000 colonoscopies in the UK Lancet 2000; 355: 1211-4.

25. Conio M, Caroli-Bosc FX, Filiberti R, et al. Endoscopic Nd: YAG laser therapy for villous adenomas of the right colon. Gastrointest Endosc 1999; 49: 504-8.

26. Tanaka S, Haruma K, Oka S, et al. Clinicopathologic features and endoscopic treatment of superficially spreading colorectal neoplasms larger than $20 \mathrm{~mm}$. Gastrointest Endosc 2001; 54: 62-6.

27. Ahmad NA, Kochman ML, Long WB, et al. Efficacy, safety, and clinical outcomes of endoscopic mucosal resection: a study of $101 \mathrm{ca}-$ ses. Gastrointest Endosc 2002: 55: 390-6.

28. Schmeck-Lindenau HJ, Kurtz W, Heine M. Inflammatory polyps: an unreported side effect of argon plasma coagulation. Endoscopy 1998; 30 (8): s93-4.

\title{
Seguridad y eficacia de la ablación de pólipos colorrectales con argón plasma
}

\author{
A. García, O. Núñez, C. González-Asanza, A. Parera, L. Menchén, C. Ripoll, C. Senent, E. Cos y \\ P. Menchén
}

Sección de Endoscopia. Servicio de Digestivo. Hospital General Universitario Gregorio Marañón. Madrid.

\section{RESUMEN}

Introducción: el argón plasma (AP), como terapia endoscópica, ha sido utilizado en diferentes lesiones digestivas, incluyendo la ablación de tejido adenomatoso de pólipos colorrectales. El objetivo del presente estudio fue evaluar la seguridad y efectividad del tratamiento con AP de pólipos colorrectales.
Material y métodos: se valoró de forma prospectiva la aplicación en la práctica clínica de AP en el tratamiento de 22 pacientes con pólipos colorrectales, de los cuales 11 pacientes con pólipos de gran tamaño fueron tratados con polipectomía endoscópica fragmentada más ablación del tejido adenomatoso con AP, y los otros 11 pacientes, con pólipos planos o en alfombra, solamente con AP. La extensión inicial 
de tejido adenomatoso a tratar tenía un diámetro medio de 22 (20-40) mm.

Resultados: la edad media de los pacientes era de 70 años. Las localizaciones más frecuentes de los pólipos fueron recto (50\%) y ciego (23\%). Se observó respuesta completa al tratamiento endoscópico de pólipos colorrectales con AP en el 90,9\% de los casos. Se produjo recurrencia en el $20 \%$, en todos los casos pólipos rectales, tras un seguimiento medio de 16,3 (8-35) meses. Todas las recurrencias fueron tratadas satisfactoriamente. No se observaron complicaciones mayores derivadas del tratamiento con AP.

Conclusiones: el tratamiento de pólipos colorrectales mediante ablación con AP es seguro y efectivo, principalmente en pólipos localizados en el colon derecho, aunque estos resultados debieran ser confirmados por estudios controlados con mayor número de pacientes.

Palabras clave: Argón plasma. Pólipos colorrectales.

\section{INTRODUCCIÓN}

La ablación o coagulación con argón plasma (AP) ha sido utilizado en la endoscopia digestiva desde 1991 cuando un electrodo específico fue diseñado para ser introducido a través del canal de trabajo del endoscopio (1). Este dispositivo electroquirúrgico monopolar no necesita del contacto con el tejido para la transmisión de una corriente eléctrica de alta energía a través del gas argón ionizado. El AP produce una quemadura térmica superficial de unos 2 a $3 \mathrm{~mm}$ de profundidad dependiendo de la energía de salida, y el flujo y tiempo aplicados (2).

El AP como terapia endoscópica ha sido utilizado en diferentes patologías como las lesiones vasculares difusas o localizadas tipo angiodisplasias o el estómago "en sandía" (3,4), el esófago de Barrett (5-8), la colitis rádica (9-11), la hemorragia por úlcera péptica $(4,12)$, el divertículo de Zenker (13), el tratamiento de tumores malignos y especialmente en la repermeabilización de prótesis metálicas, colocadas con indicación paliativa, con crecimiento tumoral intraluminal del cáncer esofágico (14-16) y los pólipos cololorrectales $(3,4)$.

Los pólipos sesiles de gran tamaño presentan con mayor frecuencia focos de neoplasia, con una gran tasa de recurrencia tras su resección endoscópica en series previas (16-46\%) al igual que una mayor incidencia de complicaciones al realizar la polipectomía, como sangrado y perforación (17-19). Por este motivo, se han buscado otros procedimientos, generalmente aplicados junto a la polipectomía, que destruyan el tejido adenomatoso residual con una menor tasa de complicaciones. En este sentido, existen estudios publicados sobre la aplicación del AP en el tratamiento del tejido residual tras polipectomía endoscópica $(2,20-22)$.

El objetivo de este estudio fue analizar la efectividad en nuestra Unidad de Endoscopia de la ablación de pólipos sesiles y planos, con o sin polipectomía fragmentada previa, y evaluar la recurrencia del tejido adenomatoso a largo plazo, así como la seguridad en su utilización en la práctica clínica.

\section{MATERIAL Y MÉTODOS}

Se diagnosticaron y fueron tratados con AP, mediante colonoscopia, 35 pacientes consecutivos con pólipos sesiles de gran tamaño o planos desde mayo de 1997 hasta diciembre de 2000. De estos pacientes, fueron excluidos del análisis 13 pacientes por presentar displasia de alto grado en el análisis histológico del pólipo, seguimiento inferior a 6 meses o pérdida durante el seguimiento.

Todos los procedimientos se realizaron dentro de la práctica clínica diaria en pacientes que acudían a realizarse la exploración de forma ambulatoria. Para la preparación del colon se utilizó solución oral con polietilenglicol y de forma ocasional la aplicación de enemas. Cuando fue necesario, por mala tolerancia del paciente, se realizó la exploración bajo sedación con propofol intravenoso administrado y controlado por un anestesista. La exploración era realizada bajo monitorización con pulsioxímetro en todos los casos. Todos los pacientes dieron su consentimiento por escrito tanto para la colonoscopia como para la aplicación de AP para el tratamiento de los pólipos colorrectales diagnosticados.

El AP era aplicado a través de un dispositivo específico a un flujo de $2,5 \mathrm{l} / \mathrm{min}$ y una potencia variable entre 40 y $80 \mathrm{~W}$ según la localización del pólipo. El tratamiento con AP fue realizado mediante colonoscopio convencional (Fujinon 200, Omiya, Japón). La polipectomía endoscópica fragmentada se realizó con un asa de polipectomía convencional y transmisión de una energía de electrocoagulación, con mezcla prefijada de coagulación y corte (Endocut; ERBE ICC 200; ERBE Electromedizin, Tübingen, Germany). La extensión del tejido adenomatoso y el tamaño de los pólipos se midió por comparación con el tamaño de una pinza de biopsia estándar abierta.

El tratamiento con AP se programó cada 15 días hasta la desaparición completa del tejido adenomatoso. Las revisiones endoscópicas se plantearon de forma que la primera era realizada a los 6 meses y después anualmente. En cada revisión se tomaron biopsias endoscópicas de la zona de resección del pólipo colorrectal, hubiera o no tejido adenomatoso visible macroscópicamente. Las exploraciones eran grabadas de tal forma que el pólipo fuera fácilmente localizado para las revisiones siguientes. Todas las exploraciones fueron realizadas por dos médicos endoscopistas, siendo siempre uno de ellos un endoscopista "senior" con experiencia en la aplicación de AP (CCA, CS, PM).

El tratamiento endoscópico de los pólipos colorrectales fue en 11 pacientes, con 11 pólipos planos o adenomas "en alfombra", tratamiento ablativo con AP como único tratamiento aplicado. En los otros 11 pacientes, el 
tratamiento con AP fue aplicado para completar el tratamiento del tejido adenomatoso residual tras polipectomía fragmentada de 11 pólipos sesiles de gran tamaño. La definición de pólipos planos se basó en criterios previamente propuestos y publicados $(23,24)$. En aquellos pólipos tratados únicamente con ablación con AP se tomaron biopsias previas con pinza fría. Este material, junto al resecado mediante polipectomía endoscópica en pólipos sesiles de gran tamaño, fue destinado para el análisis histológico.

Se definió como respuesta completa la ausencia de tejido adenomatoso macroscópico y en las muestras de biopsia endoscópica tomadas en la primera revisión tras el tratamiento. Se consideró recurrencia, en pacientes con respuesta completa previa, cuando existía de nuevo tejido adenomatoso en las áreas tratadas. El fracaso de tratamiento se consideró si persistía tejido adenomatoso visible tras tres sesiones de tratamiento con AP.

Se definieron como complicaciones graves la presencia de sangrado importante que requería de tratamiento endoscópico añadido o transfusión sanguínea, y la perforación diagnosticada en el mismo acto endoscópico o posteriormente, confirmada por pruebas radiológicas.

Las variables cualitativas se expresan en porcentajes y fueron comparadas mediante el test exacto de Fisher; y las variables cuantitativas mediante su media y rango y el test de la t de Student. Se consideró que existía significación estadística cuando $\mathrm{p}<0,05$. Para el análisis estadístico se utilizó el paquete estadístico SPSS® versión 10.0.

\section{RESULTADOS}

Veintidós pacientes fueron incluidos en el estudio con un seguimiento medio de 15 (6-35) meses. La localización más frecuente de los pólipos diagnosticados fue la rectal $(50 \%)$. Las características de los pacientes se resumen en la tabla I.

\section{Tabla I. Características basales pacientes}

\begin{tabular}{lc}
\hline Sexo (\%) & $14(64)$ \\
Hombre & $8(36)$ \\
Mujer & $70(57-83)$ \\
Edad (años) & $22(20-40)$ \\
Tamaño del tejido adenomatoso $(\mathrm{mm})$ & \\
Localización (\%) & $11(50)$ \\
Recto & $5(22,7)$ \\
Ciego & $4(18,2)$ \\
Colon izquierdo & $2(9,1)$ \\
Colon transverso & $1,7(1-3)$ \\
Sesiones & $15(6-35)$ \\
Seguimiento (meses) & \\
Técnica $n^{\circ}$ (\%) & $11(50)$ \\
AP & $11(50)$ \\
AP y polipectomía & \\
\hline
\end{tabular}

En el examen histológico se observó componente velloso en el $86 \%$ de los pólipos y displasia leve o moderada en el $81 \%$.

Se obtuvo respuesta completa en el 90,9\% de los pólipos tratados, en los cuales se aplicó una media de 1,7 (1-3) sesiones. En dos pacientes no se alcanzó respuesta tras 3 sesiones de AP por lo que fueron considerados como fracaso del tratamiento. Uno de estos pólipos estaba en recto y presentaba un diámetro de $20 \mathrm{~mm}$ y fue tratado únicamente con AP. El otro paciente tenía un pólipo sesil de $4 \mathrm{~cm}$ que fue inicialmente tratado con polipectomía fragmentada y el remanente de tejido adenomatoso de unos $20 \mathrm{~mm}$ de diámetro fue tratado con AP. En ambos casos eran pólipos vellosos con displasia leve en el análisis histológico.

Se observó recurrencia en 4 pacientes con respuesta completa $(20 \%)$ después de un periodo de seguimiento medio de 16,3 (8-35) meses. Todas las recurrencias fueron en pólipos rectales, de los cuales 3 casos fueron tratados satisfactoriamente con AP y/o polipectomía endoscópica y un caso fue sometido a resección quirúrgica endoanal.

No se objetivó relación entre la presencia de recurrencia y polipectomía fragmentada previa. Un paciente (10\%) de los tratados con AP y con respuesta completa, y 3 (30\%) de tratamiento con AP del tejido adenomatoso residual tras polipectomía fragmentada presentaron recurrencia. Sí se observó relación entre la recurrencia y el tamaño del área de tejido adenomatoso a tratar con AP $(23,8 \pm 7,5 \mathrm{~mm}$ y $13,2 \pm 7 \mathrm{~mm}, \mathrm{p}=0,01)$.

No se detectaron complicaciones mayores asociadas a la técnica. En un paciente se observó el desarrollo de pólipos inflamatorios en la región tratada, que estaba a nivel cecal, que desaparecieron en revisiones posteriores. Dos pacientes presentaron dolor abdominal tras el tratamiento que fue manejado de manera ambulatoria.

\section{DISCUSIÓN}

La coagulación con AP está basada en la transmisión de una corriente de gas argón de alta frecuencia que produce un daño térmico en la mucosa sobre la que se aplica. $\mathrm{Al}$ igual que en otras múltiples indicaciones en las que se utiliza (3-16), la base racional en el tratamiento de pólipos colorrectales es la ablación del tejido adenomatoso residual tras polipectomía o la realización de hemostasia de la base del pólipo tras la misma. En este sentido, Zlatanic y cols. (20) estudiaron la recurrencia de tejido adenomatoso tras la polipectomía fragmentada de pólipos de colon de gran tamaño. Los autores describieron la recurrencia según hubiera quedado tejido adenomatoso tras tratamiento con polipectomía endoscópica, se hubiera tratado además con AP o cuando el endoscopista consideraba que todo el tejido adenomatoso había sido resecado, siendo esta del 100, 50 y 46\%, respectivamente. En un estudio controlado más reciente, con el objetivo de estudiar la prevención de la recurrencia con AP tras polipectomía endoscópica (21), los autores observaron que el 
AP aplicado tras el tratamiento endoscópico reducía la recurrencia, describiéndose una recurrencia en 6 de 13 pacientes a los 3 meses. Otro trabajo publicado únicamente en formato resumen (22), describe una recurrencia del tejido adenomatoso en el 3,5\% de los pólipos con un periodo de seguimiento de 6 meses. En todos los estudios se sugiere la eficacia y seguridad del AP en el tratamiento de los pólipos de colon.

En nuestro estudio utilizamos el AP en dos situaciones, como único tratamiento cuando existen pólipos planos o en alfombra y tras polipectomía fragmentada para la ablación del tejido adenomatoso residual. Se observó un porcentaje de recurrencia del $20 \%$ tras un seguimiento medio de 16 meses, aproximadamente. Este porcentaje se encuentra dentro de lo descrito, entre el 3,5 y el $46 \%$, aunque nuestro periodo de seguimiento es más amplio en comparación con los estudios hasta hoy día publicados. Todos los casos de recurrencia excepto uno fueron tratados adecuadamente con terapéutica endoscópica.

La aplicación del AP como tratamiento único en pólipos adenomatosos planos o en alfombra no determinó una mayor recurrencia ni de fracaso de tratamiento, respecto a aquellos pólipos manejados inicialmente con polipectomía fragmentada y realizado el AP sobre el tejido adenomatoso residual, si bien la recurrencia estuvo en relación con el tamaño de la lesión a tratar. Este hallazgo puede tener gran relevancia en la práctica clínica, pues pólipos adenomatosos de colon derecho y ciego, donde la pared intestinal es más fina y por tanto más susceptible a la perforación, podrían ser tratados con una técnica más segura que la polipectomía. En nuestro estudio, el $23 \%$ de los pólipos tratados se localizaban en ciego y su tratamiento con AP no se siguió de complicaciones mayores en ningún caso.

Otras técnicas como la fotocoagulación con láser (25) o la mucosectomía endoscópica $(26,27)$ han sido utilizadas con buenos resultados en el tratamiento de pólipos de colon, pero la ablación con AP es una técnica más sencilla y con menos complicaciones, por lo que debería tener mayor difusión y utilización para este propósito, aunque esta sugerencia debe ser resuelta mediante estudios aleatorizados y controlados que comparen estas modalidades de tratamiento endoscópico de pólipos adenomatosos colorrectales.

La presencia de pólipos inflamatorios sobre un área previamente tratada con $\mathrm{AP}$ ha sido descrita previamente a nivel del tracto digestivo superior (28). En un caso nuestro, se observó tras la aplicación del AP en ciego, y desaparecieron espontáneamente en controles posteriores.

Concluimos, por tanto, que la aplicación de AP para el tratamiento de pólipos colorrectales es segura y efectiva, siendo esto especialmente importante en los pólipos localizados en el colon derecho, aunque estos resultados deben ser confirmados en estudios controlados con mayor número de pacientes. 